Communications in Physics, Vol. 24, No. 4 (2014), pp. 313-332

DOI:10.15625/0868-3166/24/4/4850

\title{
FOUNDATIONS OF SUPERLUMINAL RELATIVISTIC MECHANICS
}

\author{
MOHAMED E. HASSANI \\ Institute for Fundamental Research, BP.197, CTR, Ghardaïa 47800, Algeria \\ E-mail: hassani641@gmail.com \\ Received 05 September 2014 \\ Accepted for publication 12 November 2014
}

\begin{abstract}
The paper provides an elementary derivation of new superluminal spatio-temporal transformations based on the idea that, conceptually and kinematically, each subluminal, luminal and/or superluminal inertial reference frame has, in addition to its relative velocity, its proper specific kinematical parameter, which having the physical dimensions of a constant speed. Consequently, the relativity principle and causality principle both are coherently extended to superluminal velocities and, more importantly, this original approach constitutes the first basic step toward the formulation of superluminal relativistic mechanics, which is in fact a pure superluminalization of special relativity theory.
\end{abstract}

Keywords: special relativity theory; superluminal inertial reference frames; superluminal spatio-temporal transformations; superluminal relativistic mechanics.

\section{INTRODUCTION}

Special relativity theory (SRT) is among the most fundamental and well verified of all physical theories, and it is well recognized to provide an accurate description of relativistic physical phenomena. That's why it is now a basic tool of theoretical and experimental physics particularly for particle accelerators/colliders.

However, in spite of their remarkable success in their proper domain of applications for relativistic velocities $v<c$, SRT-formulae exhibit some singularity when $v=c$. In order to avoid this singularity, one can simply prohibit the existence of luminal inertial reference frames (IRFs), that is to say, a set of inertial frames that may be in rectilinear uniform motion at luminal velocity relative to each other. But such a prohibition seems to be entirely unreasonable because in the Nature; none can prevent any free material body from reaching or exceeding light speed in vacuum.

Thus, our principal motivation behind the present work is to provide a crucial elementary derivation of new superluminal spatio-temporal transformations (STs) based on the idea that, conceptually and kinematically, each subluminal $(0 \leq v<c)$, luminal $(v=c)$ and/or superluminal $(v>c)$ inertial reference frame has, in addition to its relative velocity, its proper specific kinematical parameter (SKP), which having the physical dimensions of a constant speed. As a direct consequence, STs should allow us to extend SRT to luminal and superluminal IRFs without singularity.

(C)2014 Vietnam Academy of Science and Technology 
Explicitly, we are dealing with the superluminalization of SRT that leads directly to superluminal relativistic mechanics in which the theoretical maximal possible velocity of an ordinary massive particle or of a physical signal is not necessarily equal to the light speed in vacuum but can be higher than $c$ as we will see later. This means the light speed, $c=299792458 \mathrm{~ms}^{-1}$, is limiting speed only in the context of SRT because the Lorentz transformation (LT), which is the core of SRT, becomes meaningless when the relative velocity of IRF reaches or exceeds the light speed, that is when $v \geq c$ Lorentz factor $\gamma=\left(1-v^{2} / c^{2}\right)^{-1 / 2}$ becomes imaginary or infinite.

\section{CAUSALITY PRINCIPLE}

The assumption that information traveling faster than light speed in vacuum represents a violation of causality principle is only valid in the context of SRT because of LT, which is exclusively applicable to IRFs in relative uniform motion with subluminal velocities.

Nevertheless, causality simply means that the cause of an event precedes the effect of the event. In this case, e.g., a massive particle is emitted before it is absorbed in a detector. If the particle's velocity was one trillion times faster than $c$, the cause would still precede the effect, and causality principle would not be violated since, here, LT should be replaced with STs because the particle in question was moving in superluminal space-time not in Minkowski space-time. Therefore, in superluminal space-time, the superluminal signals do not violate the causality principle but they can shorten the luminal vacuum time span between cause and effect.

Theoretically, SRT does not prohibit the existence of superluminal velocities because a deeply critical reading of Einstein's papers on SRT has already shown more conclusively that Einstein himself used, at the same time, the subluminal and superluminal velocities in SRT. For example, in his 1905' paper [1], he wrote: '... Taking into consideration the principle of constancy of the velocity of light we find that

$$
t_{B}-t_{A}=\frac{r_{A B}}{c-v} \text { and } t_{A}^{\prime}-t_{B}=\frac{r_{A B}}{c+v}
$$

where $r_{A B}$ denotes the length of the moving rod-measured in the stationary system ...'

It is quite clear from the above equations, that is, since in Einstein's paper $v(v<c)$ is the relative velocity between the two IRFs, $K$ and $K^{\prime}$, thus $c-v$ and $c+v$ are subluminal and superluminal velocity respectively. Therefore, outside the SRT-context, none can forbid the existence of superluminal velocities in the real physical world.

\section{SUPERLUMINAL FORMALISM}

The theory here to be developed is based -like any physical model - on its proper principles as basis for organizing and facilitating our understanding of its internal structure and external consequences. The central elements that constitute the core of the present work, which will henceforth be called 'superluminal relativistic mechanics' are the principle of relative motion, the principle of kinematical levels, the superluminal space-time (geometry) and the superluminal spatio-temporal transformations (STs).

\section{III.1. Principle of relative motion}

If the inertial reference frame (IRF) $S^{\prime}$ moves in straight-line at a constant velocity $v$ relative to the (IRF) $S$, then $S$ moves in straight-line at a constant velocity $-v$ relative to $S^{\prime}$. 


\section{III.2. Principle of kinematical levels}

Conceptually, there are three kinematical levels (KLs) namely subluminal, luminal and superluminal level, such that:

i) Each KL is characterized by a set of IRFs moving with respect to each other at a constant subluminal velocity $0 \leq v<c$ ) in the first KL; at a constant luminal velocity $(v=c)$ in the second KL and at a constant superluminal velocity $(v>c)$ in the third KL.

ii) Each IRF has, in addition to its relative velocity of magnitude $v$, its proper specific kinematical parameter (SKP), which having the physical dimensions of a constant speed defined as

$$
\left\{\begin{array}{l}
\vartheta(v)=c, 0 \leq v<c \\
\vartheta(v)>v, c \leq v<\infty \\
\vartheta^{2}(-v)=\vartheta^{2}(v), \forall v
\end{array} .\right.
$$

iii) All the subluminal IRFs are linked with each other via Galilean transformation and/or LT.

iv) All the luminal IRFs are linked with each other via luminal (spatio-temporal) transformations.

v) All the superluminal IRFs are linked with each other via STs.

vi) All the IRFs belonging to the same KL are equivalent.

These two principles suffice - with the superluminal space-time (geometry) and STs - for the attainment of a simple and consistent theory of superluminal relativistic mechanics, which should understand as an extension of SRT to luminal and superluminal IRFs. Here, the above adjective 'relativistic' is strictly speaking, relating to the Galilean relativity principle and its Poincaré-Einstein extension.

\section{III.3. Superluminal space-time}

Since, conceptually, SRT does not forbid the existence of superluminal motions and, as we have already seen, Einstein himself employed, simultaneously, the subluminal and superluminal velocities in his 1905' paper [1], therefore, the question arises naturally: what is the appropriate geometry of space-time to describe superluminal physical phenomena?

In order to answer adequately the above question, we shall take into account the principle of KLs, more precisely, the definition of SKP (1). Hence, we can undertake to establish the mathematical structure of superluminal space-time theoretically inspired from the existence of superluminal motions. The said mathematical structure of superluminal space-time as a seat of superluminal physical phenomena should be defined by the following superluminal quadratic form (superluminal metric)

$$
x^{\prime 2}+y^{\prime 2}+z^{\prime 2}-\vartheta^{2}(v) t^{\prime 2}=x^{2}+y^{2}+z^{2}-\vartheta^{2}(v) t^{2}
$$

Further, according to the definition of SKP (1), the superluminal quadratic form (2) may be reduced to that of Minkowski. The signature $(+,+,+,-)$ into (2) implies that the geometry of superluminal space-time is not completely Euclidean, it is in fact non-Euclidean because as we will see later in superluminal regime, space 'contracts' and time 'dilates' as in Minkowski space-time in subluminal KL for relativistic velocities . 
Therefore, According to the principle of relative motion, the superluminal quadratic form (2) should be invariant under certain superluminal spatio-temporal transformations during any transition from a superluminal IRF to another. For this reason, we can also define a superluminal four-vector of position as follows: relatively to (IRF) $S$, we call superluminal four-vector of position of a superluminal event of spatio-temporal coordinates $(x, y, z, t)$, a vector $\mathbf{R}$ of components:

$$
\left(x_{1}=x, x_{2}=y, x_{3}=z, x_{4}=i \vartheta(v) t\right), \quad i=\sqrt{-1} .
$$

\section{III.4. Superluminal spatio-temporal transformations}

The superluminal (spatio-temporal) transformations, the superluminal IRFs and the hypothetical tachyons as superluminal particles have a long history and these concepts were generally related to SRT. However, the tangible evidence of superluminal motions is a fundamental question debated in modern physics.

Investigations on tachyonic motion and superluminal transformations inaugurated additional opportunities which were studied by many researchers, for instance, by Bilaniuk and Sudarshan [2], Recami [3], Mignani [4], Patty [5], Recami and Maccarrone [6], Parker [7], Marchildon, Antippa and Everett [8], Sutherland and Shepanski [9].

Thus, with the help of the principle of relative motion and the principle of KLs, we undertake to derive the superluminal spatio-temporal transformations (STs) for spatio-temporal coordinates, so that the STs should satisfy the following principal requirements:

a) The STs should ensure the invariance of the superluminal quadratic form (2).

b) The STs should be real.

c) The STs should be linear.

d) The STs should have an algebraic structure of an orthogonal-orthochronous group ${ }^{1}$.

By taking into account the principle of KLs, we can derive the expected STs in simple manner. To this end, let us consider two IRFs $S$ and $S^{\prime}$, which are in relative uniform translational motion at superluminal velocity $\mathbf{v}$ of magnitude $v$ such that $c<v<\vartheta(v)$. Further, let us assume that a superluminal event can be characterized by spatio-temporal coordinates $(x, y, z, t)$ in $S$ and $\left(x^{\prime}, y^{\prime}, z^{\prime}, t^{\prime}\right)$ in $S^{\prime}$.

To simplify the algebra let the relative superluminal velocity vector $v$ of IRFs be along their common $x \mid x^{\prime}$-axis with corresponding parallel planes. Also, the two origins $\mathrm{O}$ and $\mathrm{O}$ ' coincide at the moment $t=t^{\prime}=0$ (henceforth, two superluminal IRFs related in this way are said to be in the standard configuration). The supposed homogeneity and isotropy of space and uniformity of time in all superluminal IRFs require that the STs must be real and linear so that the simplest form they can take (when for example the transition operated from $S$ to $S^{\prime}$ ) is:

$$
S \rightarrow S^{\prime}:\left\{\begin{array}{l}
x^{\prime}=\eta(x-v t) \\
y^{\prime}=y \\
z^{\prime}=z \\
t^{\prime}=\lambda x+\zeta t
\end{array} .\right.
$$

In order to determine the expressions of the coefficients $\eta, \lambda$ and $\zeta$ we must use the idea of the homogeneity and isotropy of space and uniformity of time in all superluminal IRFs, and

\footnotetext{
${ }^{1}$ That is to say the notion of past, present and future is preserved and this implies, among other things, the preservation of the causality principle in all the IRFs.
} 
the requirement (a). Therefore, when Eqs.(3) are substituted in left-hand side of the superluminal quadratic form (2), we get

$$
\eta^{2}(x-v t)^{2}+y^{2}+z^{2}-\vartheta^{2}(v) \cdot(\lambda x+\zeta t)^{2}=x^{2}+y^{2}+z^{2}-\vartheta^{2}(v) t^{2} .
$$

From which we have

$$
\left\{\begin{array}{l}
\eta^{2}-\lambda^{2} \vartheta^{2}(v)=1 \\
\eta^{2} v+\lambda \zeta \vartheta^{2}(v)=0 \\
\zeta^{2} \vartheta^{2}(v)-\eta^{2} v^{2}=\vartheta^{2}(v)
\end{array}\right.
$$

The system of three Eqs. (5) when solved for $\eta, \lambda$ and $\zeta$ yields

$$
\eta=1 / \sqrt{1-v^{2} / \vartheta^{2}(v)} ; \lambda=-\left[v / \vartheta^{2}(v)\right] / \sqrt{1-v^{2} / \vartheta^{2}(v)} ; \quad \zeta=1 / \sqrt{1-v^{2} / \vartheta^{2}(v)}
$$

Now, by substituting (6) in (3), we obtain the expressions of the expected STs, i.e., ST and its inverse $(\mathrm{ST})^{-1}$ :

$$
S \rightarrow S^{\prime}:\left\{\begin{array}{l}
x^{\prime}=\eta(x-v t) \\
y^{\prime}=y \\
z^{\prime}=z \\
t^{\prime}=\eta\left(t-\frac{v x}{\vartheta^{2}(v)}\right)
\end{array}\right.
$$

and

$$
S^{\prime} \rightarrow S:\left\{\begin{array}{l}
x=\eta\left(x^{\prime}+v t^{\prime}\right) \\
y=y^{\prime} \\
z=z^{\prime} \\
t=\eta\left(t^{\prime}+\frac{v x^{\prime}}{\vartheta^{2}(v)}\right)
\end{array}\right.
$$

where

$$
\eta=1 / \sqrt{1-v^{2} / \vartheta^{2}(v)} \text { and } c<v<\vartheta(v)
$$

Furthermore, we can make sure that the STs preserve really the invariance of superluminal quadratic form (2) during, e.g., any transition from $S$ to $S^{\prime}$. With this aim, we have

$$
\begin{array}{r}
x^{\prime 2}+y^{\prime 2}+z^{\prime 2}-\vartheta^{2}(v) t^{\prime 2}=\eta^{2}(x-v t)^{2+} y^{2}+z^{2}-\vartheta^{2}(v) \eta^{2}\left(t-v x / \vartheta^{2}(v)\right)^{2} \\
=\eta^{2}\left(1-v^{2} / \vartheta^{2}(v)\right) x^{2+} y^{2}+z^{2}-\vartheta^{2}(v) \eta^{2}\left(1-v^{2} / \vartheta^{2}(v)\right) t^{2} \\
=x^{2}+y^{2}+z^{2}-\vartheta^{2}(v) t^{2}
\end{array}
$$

This is in good agreement with the principle of relative motion and the principle of KLs, also it is easy to verify that the STs (7) and (8) which depending on the parameters $v$ and $\vartheta(v)$ form a linear orthogonal-orthochronous group since their determinant is equal to +1 . Therefore, the STs satisfy all the imposed requirements (a), (b), (c) and (d). From all that, we can logically affirm that the relativity principle and the causality principle are extended to superluminal IRFs via STs. In arriving at this conclusion, we can assert to have really established the basic foundations for the superluminal relativistic mechanics. 


\section{III.5. Luminal spatio-temporal transformations}

We begin this subsection by the following quote from Dirac's Noble Lecture (12 December $1933)$ entitled 'Theory of electrons and positrons': "The variables $\alpha$ also give rise to some rather unexpected phenomena concerning the motion of the electron. These have been fully worked out by Schrödinger. It is found that an electron which seems to us to be moving slowly, must actually have a very high frequency oscillatory motion of small amplitude superposed on the regular motion which appears to us. As a result of this oscillatory motion, the velocity of the electron at any time equals the velocity of light. This is a prediction which cannot be directly verified by experiment, since the frequency of the oscillatory motion is so high and its amplitude is so small. But one must believe in this consequence of the theory, since other consequences of the theory which are inseparably bound up with this one, such as the law of scattering of light by an electron, are confirmed by experiment."

This passage reflects the theoretical limit of validity of SRT via LT because, here, the electron as a fundamental elementary particle has well-known mass and charge, and its oscillatory motion at the velocity of light implies that such a luminal oscillatory motion should manifest in the (super-) luminal space-time as a seat of (super-) luminal physical phenomena.

Furthermore, since each physical object has its proper IRF, consequently, the electron should have its proper IRF. In this sense, the existence of luminal IRFs constitutes the upper limit of validity of LT and SRT. The luminal spatio-temporal transformations that ensure the link between all the luminal IRFs are in fact a special case of STs, explicitly, according to the definition of SKP (1), STs (7) and (8) reduce to the luminal spatio-temporal transformations for the case $v=c$. That is to say, when the IRFs $S$ and $S^{\prime}$ are in relative motion at luminal velocity of magnitude $c$ with respect to each other.

\section{SUPERLUMINAL RELATIVISTIC MECHANICS}

As already mentioned, before the present investigation, there was lots of research works appeared in the superluminal/tachyonic literature for almost fifty years and focalized on the possibility of extending SRT through the generalization of LT to superluminal IRFs.

In view of the fact that our main aim is the superluminalization of SRT as a first step toward the superluminal relativistic mechanics (SupRelMec), therefore, the SRT-effects should exist in the present superluminal formalism. SupRelMec comprises two principal parts, namely the superluminal relativistic kinematics and the superluminal relativistic dynamics.

\section{IV.1. Superluminal relativistic kinematics}

In what follows it is endeavored to establish the formulation of the superluminal relativistic kinematics which should drew from STs. We will show the existence of the superluminal relativistic length contraction and time dilation; we will derive the transformations of superluminal velocities and accelerations. Once again, we will see, in the context of superluminal relativistic kinematics, that the causality principle is at the same time conserved and extended to superluminal velocities without any contradiction because, in superluminal space-time, the superluminal velocities do not violate the causality but they can only shorten the luminal vacuum time span between cause and effect. This is the main property of the superluminal space-time as a seat of superluminal physical phenomena. 


\section{IV.1.1. Superluminal relativistic length contraction}

Suppose two superluminal IRFs $S$ and $S^{\prime}$ in standard configuration and consider a body at relative rest with respect to $S^{\prime}$. Let $\left(x_{1}^{\prime}, y_{1}^{\prime}, z_{1}^{\prime}\right)$ and $\left(x_{2}^{\prime}, y_{2}^{\prime}, z_{2}^{\prime}\right)$ be the coordinates of its material points referred to $S^{\prime}$. Between the coordinates $\left(x_{1}, y_{1}, z_{1}\right)$ and $\left(x_{2}, y_{2}, z_{2}\right)$ of these material points relative to $S$, there obtain at each time $t$ of $S$, according to STs (7) and (8), the relations

$$
x_{2}-x_{1}=\left(x_{2}^{\prime}-x_{1}^{\prime}\right) \sqrt{1-v^{2} / \vartheta^{2}(v)} ; y_{2}-y_{1}=y_{2}^{\prime}-y_{1}^{\prime} ; z_{2}-z_{1}=z_{2}^{\prime}-z_{1}^{\prime} .
$$

Or simply by taking account of the direction of superluminal relative motion, i.e., IRFs common $x \mid x$-axis, the expected superluminal relativistic length contraction formula may be written as

$$
\Delta x=\Delta x^{\prime} \sqrt{1-v^{2} / \vartheta^{2}(v)}
$$

where $\Delta x^{\prime}$ is the proper length of a material body in state of relative rest in $S^{\prime}$.

Result: in superluminal relativistic kinematics, the kinematic shape of material body supposed to be in a state of uniform translation depends thus on its superluminal velocity relative to IRF, namely by differing from its proper geometric shape in being contracted in the direction of superluminal relative motion.

\section{IV.1.2. Superluminal relativistic time dilation}

Like before, Suppose two superluminal IRFs $S$ and $S^{\prime}$ in standard configuration and assume that there is a clock at relative rest at the origin of coordinates of $S^{\prime}$, which runs $v_{0}$ times faster than the clocks used in the two IRFs $S$ and $S^{\prime}$ for the measurement of time,i.e., this clock executes $v_{0}$ periods during a time in which the reading of a clock which is at relative rest with respect to it and is of the same nature of the other clocks used in $S$ and $S^{\prime}$ for the measurement of time, increases by one unit. Question: How fast does the first mentioned clock run as viewed from $S$ ?

From above considerations and with the help of STs, we obtain the expected superluminal relativistic time dilation formula

$$
\Delta t=\Delta t^{\prime} / \sqrt{1-v^{2} / \vartheta^{2}(v)} .
$$

Result: In superluminal relativistic kinematics, a clock moving uniformly with superluminal velocity relative to IRF, runs when viewed from that frame, more slowly than the same clock when is at relative rest with respect to this frame. Furthermore, in terms of frequency, we get from (11) the following important formula

$$
v=v^{\prime} \sqrt{1-v^{2} / \vartheta^{2}(v)}
$$

The formula (12) may be physically interpreted as a superluminal transverse relativistic Doppler effect and consequently should admit of a very interesting application, particularly, to the powerful radio quasars that exhibit the well-known superluminal motions. Furthermore, it is easy to show that the formula (12) is in fact a special case of the superluminal Doppler effect

$$
v=v^{\prime} \frac{\sqrt{1-v^{2} / \vartheta^{2}(v)}}{1+\frac{v}{\vartheta(v)} \cos \theta} .
$$


Here, $v^{\prime}$ is the frequency of a light source when referred to a comoving IRF, moves so that the connecting line -light source-observer-forms the angle $\theta$ with the velocity of the light source, referred to an IRF at relative rest with respect to the observer, then the frequency $v$ perceived by the observer is given by formula (13). In the case when $\theta=\pi / 2$, the light was emitted at the moment of closest approach, and one obtains the transverse superluminal Doppler effect (12).

\section{IV.1.3. Transformations of superluminal velocities}

Let us call the vector $\mathbf{u}\left(u_{x}, u_{y}, u_{z}\right)$ of magnitude $u$ the superluminal velocity vector of a material point in (IRF) $S$ such that $c<u<\vartheta(v)$, and let us consider a second (IRF) $S^{\prime}$ in straightline uniform motion at superluminal velocity of magnitude $v$ relative to $S$ along the $x$-axis. In $S^{\prime}$ the same material point is characterized by the superluminal velocity vector $\mathbf{u}^{\prime}\left(u_{x^{\prime}}^{\prime}, u_{y^{\prime}}^{\prime}, u_{z^{\prime}}^{\prime}\right)$ of magnitude $u^{\prime}$ with $c<u^{\prime}<\vartheta(v)$. The two frames $S$ and $S^{\prime}$ are connected by STs. Thus, a direct differentiation of ST (7), gives the required transformations of superluminal velocities:

$$
S \rightarrow S^{\prime}:\left\{\begin{array}{l}
u_{x^{\prime}}^{\prime}=\left(u_{x}-v\right)\left[1-\frac{u_{x} v}{\vartheta^{2}(v)}\right]^{-1} \\
u_{y^{\prime}}^{\prime}=u_{y}\left[1-\frac{v^{2}}{\vartheta^{2}(v)}\right]\left[1-\frac{u_{x} v}{\vartheta^{2}(v)}\right]^{-1}, \\
u_{z^{\prime}}^{\prime}=u_{z}\left[1-\frac{v^{2}}{\vartheta^{2}(v)}\right]\left[1-\frac{u_{x} v}{\vartheta^{2}(v)}\right]^{-1}
\end{array}\right.
$$

and

$$
S^{\prime} \rightarrow S:\left\{\begin{array}{l}
u_{x}=\left(u_{x^{\prime}}^{\prime}+v\right)\left[1+\frac{u_{x^{\prime}}^{\prime}}{\vartheta^{2}(v)}\right]^{-1} \\
u_{y}=u_{y^{\prime}}^{\prime}\left[1-\frac{v^{2}}{\vartheta^{2}(v)}\right]\left[1+\frac{u_{x^{\prime}}^{\prime}}{\vartheta^{2}(v)}\right]^{-1} . \\
u_{z}=u_{z^{\prime}}^{\prime}\left[1-\frac{v^{2}}{\vartheta^{2}(v)}\right]\left[1+\frac{u_{x^{\prime}}^{\prime} v}{\vartheta^{2}(v)}\right]^{-1}
\end{array}\right.
$$

\section{IV.1.4. Addition law of superluminal velocities}

Consider now the important particular case, that is, when the material point moves in $S$ along the $x$-axis, we obtain from (15):

$$
u_{x}=\frac{u_{x^{\prime}}^{\prime}+v}{1+\frac{u_{x^{\prime}}^{\prime} v}{\vartheta^{2}(v)}}, \quad u_{y}=0, \quad u_{z}=0 .
$$

Consequently, if we put $u_{x^{\prime}}^{\prime}=u^{\prime}$ and $u_{x}=u$, we get the following expected addition law of superluminal velocities

$$
u=\frac{u^{\prime}+v}{1+\frac{u^{\prime} v}{\vartheta^{2}(v)}}
$$

Remark: If we set $u^{\prime}=\vartheta(v)$, we obtain from (16) the following interesting property:

$$
u=\frac{\vartheta(v)+v}{1+\frac{v}{\vartheta(v)}}=\vartheta(v) .
$$

The result (17) means that the SKP, $\vartheta(v)$, is really constant in all the superluminal IRFs. 


\section{IV.1.5. Superluminal velocity four-vector}

In superluminal relativistic kinematics, we define a superluminal velocity four-vector of a material point as follows: Let the vector $\mathbf{u}\left(u_{x}, u_{y}, u_{z}\right)$ of magnitude $u=\sqrt{u_{x}^{2}+u_{y}^{2}+u_{z}^{2}}$ be the superluminal velocity vector of the material point under consideration, we call a superluminal velocity four-vector, the quantity

$$
\mathbf{U}=\frac{d \mathbf{R}}{d t^{*}}=(\eta \mathbf{u}, i \eta \vartheta(u)), \quad \eta=1 / \sqrt{1-u^{2} / \vartheta^{2}(u)},
$$

where $d t^{*}=\eta^{-1} d t$ is the proper time of material point and $\mathbf{R}=\left(x_{1}=x, x_{2}=y, x_{3}=z, x_{4}=i \vartheta(u) t\right)$ is its four-vector of position. Or more explicitly

$$
\mathbf{U}=\left(u_{1}=\eta u_{x}, u_{2}=\eta u_{y}, u_{3}=\eta u_{z}, u_{4}=i \eta \vartheta(u)\right)
$$

Also, we can remark from (19) that

$$
\mathbf{U}^{2}=-\vartheta^{2}(u)
$$

That is to say all the superluminal velocity four-vectors have a magnitude of $\vartheta(u)$. This is another expression of the fact that in superluminal relativistic kinematics, there is no absolute rest - at least- we are always moving forward through time! Moreover, according to the definition of SKP (1), it follows that even if $\mathbf{u}=\mathbf{0}$, we get $\mathbf{U}=(0,0,0, i c)$.

\section{IV.1.6. Transformations of superluminal velocity four-vector}

The superluminal velocity four-vector $\mathbf{U}$ being a superluminal four-vector, its components should be invariant under STs during any change of superluminal IRF. Let the superluminal IRFs $S$ and $S^{\prime}$ be in standard configuration. We call the vector $\mathbf{u}\left(u_{x}, u_{y}, u_{z}\right)$ of magnitude $u$ the superluminal velocity vector of a material point relative to $S$ such that $c<u<\vartheta(v)$. In $S^{\prime}$ the same material point is characterized by the superluminal velocity vector $\mathbf{u}^{\prime}\left(u_{x^{\prime}}^{\prime}, u_{y^{\prime}}^{\prime}, u_{z^{\prime}}^{\prime}\right)$ of magnitude $u^{\prime}$ with $c<u^{\prime}<\vartheta(v)$.

We have according to ST (8)

$$
S \rightarrow S^{\prime}:\left\{\begin{array}{l}
u_{1}^{\prime}=\eta_{(v)}\left(u_{1}-\varepsilon u_{0}\right) \\
u_{2}^{\prime}=u_{2} \\
u_{3}^{\prime}=u_{3} \\
u_{0}^{\prime}=\eta_{(v)}\left(u_{0}-\varepsilon u_{1}\right)
\end{array}\right.
$$

where

$$
\begin{aligned}
& \eta_{(v)}=1 / \sqrt{1-\varepsilon^{2}}, \quad \varepsilon=v / \vartheta(v), \\
& \mathbf{U}=\left(u_{1}=\eta u_{x}, u_{2}=\eta u_{y}, u_{3}=\eta u_{z}, u_{4}=i u_{0}=i \eta \vartheta(u)\right), \\
& \mathbf{U}^{\prime}=\left(u_{1}^{\prime}=\eta^{\prime} u_{x^{\prime}}^{\prime}, u_{2}^{\prime}=\eta^{\prime} u_{y^{\prime}}^{\prime}, u_{3}^{\prime}=\eta^{\prime} u_{z^{\prime}}^{\prime}, u_{4}^{\prime}=i u_{0}^{\prime}=i \eta^{\prime} \vartheta\left(u^{\prime}\right)\right),
\end{aligned}
$$

with

$$
\eta=\frac{1}{\sqrt{1-u^{2} / \vartheta^{2}\left(u^{\prime}\right)}}, \quad \eta^{\prime}=\frac{1}{\sqrt{1-u^{\prime 2} / \vartheta^{2}(u)}}
$$


By taking into account the principle of KLs or more precisely the fact that all the IRFs belonging to the same KL are equivalent thus, without loss of generality, we can consider the interesting special case that is when $\vartheta(v)=\vartheta(u)=\vartheta\left(u^{\prime}\right)$, hence from all that, we deduce

$$
S \rightarrow S^{\prime}:\left\{\begin{array}{l}
\eta^{\prime} u_{x^{\prime}}^{\prime}=\eta \eta_{(v)}\left(u_{x}-v\right) \\
\eta^{\prime} u_{y^{\prime}}^{\prime}=\eta u_{y} \\
\eta^{\prime} u_{z^{\prime}}^{\prime}=\eta u_{z} \\
\eta^{\prime} \vartheta\left(u^{\prime}\right)=\eta \eta_{(v)} \vartheta\left(u^{\prime}\right)\left(1-\frac{u_{x} v}{\vartheta^{2}(v)}\right)
\end{array} .\right.
$$

Noting, the fourth equation in above transformation gives us

$$
\frac{\eta}{\eta^{\prime}}=\frac{1}{\eta_{(v)}\left(1-\frac{u_{x} v}{\vartheta^{2}(v)}\right)} .
$$

Therefore, from where we can obtain, once again, the transformations of superluminal velocities. This means we have really defined a superluminal velocity four-vector invariant under STs during any change of IRF.

\section{IV.1.7. Transformations of superluminal accelerations}

For the superluminal acceleration of the moving material point treated previously, we have relatively to $S$ and $S^{\prime}$, respectively:

$$
\mathbf{a}=\left(a_{x}=d u_{x} / d t, a_{y}=d u_{y} / d t, a_{z}=d u_{z} / d t\right),
$$

and

$$
\mathbf{a}^{\prime}=\left(a_{x^{\prime}}^{\prime}=d u_{x^{\prime}}^{\prime} / d t^{\prime}, a_{y^{\prime}}^{\prime}=d u_{y^{\prime}}^{\prime} / d t^{\prime}, a_{z^{\prime}}^{\prime}=d u_{z^{\prime}}^{\prime} / d t^{\prime}\right) .
$$

The differentiation of the fourth equation in ST (7) gives $d t^{\prime}=\eta\left(d t-v d x / \vartheta^{2}(v)\right)$, thus we get from (14), (15), (24) and (25), the required transformations of superluminal accelerations:

$$
S \rightarrow S^{\prime}:\left\{\begin{array}{l}
a_{x^{\prime}}^{\prime}=\eta^{-3 / 2}\left[1-\frac{u_{x} v}{\vartheta^{2}(v)}\right]^{-3} a_{x} \\
a_{y^{\prime}}^{\prime}=\eta^{-1}\left[1-\frac{u_{x} v}{\vartheta^{2}(v)}\right]^{-3}\left[\left(1-\frac{u_{x} v}{\vartheta^{2}(v)}\right) a_{y}+\left(\frac{u_{y} v}{\vartheta^{2}(v)}\right) a_{x}\right], \\
a_{z^{\prime}}^{\prime}=\eta^{-1}\left[1-\frac{u_{x} v}{\vartheta^{2}(v)}\right]^{-3}\left[\left(1-\frac{u_{x} v}{\vartheta^{2}(v)}\right) a_{z}+\left(\frac{u_{z} v}{\vartheta^{2}(v)}\right) a_{x}\right]
\end{array}\right.
$$

and

$$
S^{\prime} \rightarrow S:\left\{\begin{array}{l}
a_{x}=\eta^{-3 / 2}\left[1+\frac{u_{x^{\prime}}^{\prime} v}{\vartheta^{2}(v)}\right]^{-3} a_{x^{\prime}}^{\prime} \\
a_{y}=\eta^{-1}\left[1+\frac{u_{x^{\prime}}^{\prime} v}{\vartheta^{2}(v)}\right]^{-3}\left[\left(1+\frac{u_{x^{\prime}}^{\prime} v}{\vartheta^{2}(v)}\right) a_{y^{\prime}}^{\prime}-\left(\frac{u_{y^{\prime}}^{\prime} v}{\vartheta^{2}(v)}\right) a_{x^{\prime}}^{\prime}\right], \\
a_{z^{\prime}}^{\prime}=\eta^{-1}\left[1+\frac{u_{x} v}{\vartheta^{2}(v)}\right]^{-3}\left[\left(1+\frac{u_{x^{\prime}}^{\prime} v}{\vartheta^{2}(v)}\right) a_{z^{\prime}}^{\prime}-\left(\frac{u_{z^{\prime}}^{\prime}}{\vartheta^{2}(v)}\right) a_{x^{\prime}}^{\prime}\right]
\end{array}\right.
$$

\section{IV.1.8. Superluminal accelerations four-vector}

Concerning the superluminal acceleration four-vector of the moving material point that previously treated; this quantity may be derived from the definition of superluminal velocity fourvector (18), namely 


$$
\mathbf{U}=\frac{d \mathbf{R}}{d t^{*}}=(\eta \mathbf{u}, i \eta \vartheta(u)), \quad d t^{*}=\eta^{-1} d t, \quad \eta=1 / \sqrt{1-u^{2} / \vartheta^{2}(u)} .
$$

By differentiating the above relation with respect to proper time $t^{*}$ of the material point, we get

$$
\mathbf{A}=\frac{d \mathbf{U}}{d t^{*}}
$$

Also, from relation (20), we deduce the following important property

$$
\mathbf{U}^{2}=-\vartheta^{2}(u) \Rightarrow 2 \mathbf{U} \frac{d \mathbf{U}}{d t^{*}}=0 \Rightarrow \mathbf{U} \cdot \mathbf{A}=0 .
$$

Hence, in superluminal relativistic kinematics or more precisely in superluminal spacetime, the superluminal velocity four-vector and acceleration four-vector are orthogonal.

\section{IV.1.9. Superluminal relativistic kinematics and Causality principle}

The main aim of this sub-subsection is to show, in the context of superluminal relativistic kinematics, that the causality as a well-established universal principle would be absolutely valid for subluminal, luminal and superluminal signals under any natural and/or artificial circumstances and any attempt to apply special relativity theory (SRT) to superluminality of physical phenomena would be a complete waste of time since this theory has the light speed in vacuum as an upper limiting speed in its proper validity domain of applications.

Due to the very old common misconception about the causality principle and its possible violation by (hypothetical) superluminal signal, which historically goes back to Einstein's note published in 1907 [10], many textbooks and research articles denied the real possibility of signal velocities faster than vacuum speed of light. However, there is a number of important theoretical and experimental works on the topic published in Nature; Science; Phys. Lett. Rev.; Phys. Lett. A. etc., particularly the papers [ [11-17].

Furthermore, we can mention as a concrete illustration the experimental evidence of the (apparent) superluminal expansion, which has previously been observed at astronomical distance several times [18-21]; the same superluminal expansion has also been observed at $85-95 \mathrm{~km}$ altitudes by Inan et al. [22] during their investigation of rapid lateral expansion of optical luminosity in lighting. Based on the photometer data alone, they found that the apparent speed of lateral expansion of each of the two observed luminous events is 3.10 times the light speed in a narrow altitude range $(85-95 \mathrm{~km})$.

Concerning the superluminality of light propagation, Wang et al., [17] used gain-assisted linear anomalous dispersion to observe superluminal light propagation in atomic cesium gas, their work published in Nature, the author claimed that: " The observed superluminal light pulse propagation is not at odds with causality, being a direct consequence of classical interference between its different frequency components in an anomalous dispersion region."

These facts combined with the principle of kinematical levels (KLs) allow us to suggest that the causality as a universal principle would be valid in all the KLs. Therefore, from such a suggestion we arrive, again, at the following new conception of causality itself: the validity of causality in all the KLs implies that there are conceptually three kinds of causality, viz., subluminal causality, luminal causality and superluminal causality, and each kind is characterized by its proper circumstances. Since each KL is, as we know from the principle of KLs, represented by its own 
group of spatio-temporal transformations. Thus, the main role of each group is the study of the chronology of events defined by the couple (cause, effect). This implies that we cannot study, e.g., superluminal causality with the help of the Lorentz group and also we cannot apply Galilean group to luminal causality.

Returning to our principal aim, namely, with the help of ST (7) and the definition of SKP (1), we should be able to prove -via a simple thought experiment- that the superluminal causality should have a natural existence and do not violate the "conventional causality" since, as we will see, the chronological order of events is perfectly respected.

However, in 1907, Einstein used the addition theorem of velocities (ATV) to superluminal velocities in order to prove the violation of causality. This approach is incorrect even if it is based on thought experiment (gedankenexperiment) because SRT is clearly inapplicable to superluminal motions and also because superluminal velocities as such should be defined in superluminal spacetime, not in Minkowski space-time. Hence, physically, we cannot apply ATV to superluminal signals since ATV itself is only valid for subluminal velocities, without forgetting that ATV is derived from LT, which is uniquely valid for subluminal velocities, which is why Einstein [23] had, in this case, rightly affirmed that "A relative motion of reference systems with superluminal velocity is incompatible with our principles."

-Thought Experiment: Let us consider two IRFs $S$ and $S^{\prime}$, which are in relative uniform translational motion at superluminal velocity $\mathbf{v}$ of magnitude $v$ such that $c<v<\vartheta(v)$. Further, let us assume that a superluminal event can be characterized by spatio-temporal coordinates $(x, y, z, t)$ in $S$ and $\left(x^{\prime}, y^{\prime}, z^{\prime}, t^{\prime}\right)$ in $S^{\prime}$ and the relative superluminal velocity vector $\mathbf{v}$ of IRFs be along their common $x \mid x^{\prime}$-axis with corresponding parallel planes. Also, the two origins $\mathrm{O}$ and $\mathrm{O}^{\prime}$ coincide at the moment $t=t^{\prime}=0$.

Now, imaging Alice and Bob when they were together (say, when they were coincident at $O \equiv O^{\prime}$ ) could have arranged in advance that they would send to each other a superluminal signal. Suppose Alice is sitting in her laboratory on her space station, and she sends out a superluminal signal. Let us call the event of sending the signal event $A$. Alice sends the signal to Bob, who is out on a spaceship, and he received it at event $B$.

We said that Alice sends a superluminal signal to Bob, who is on a distant spaceship. Let us call Alice's IRF $S$ and Bob's IRF $S^{\prime}$. If Bob's spaceship is traveling away from Alice at a superluminal velocity $v$ and the sending signal (event $A$ ) propagating at superluminal velocity $u$ such that $v \leq u<\vartheta(v)$. Since according to the relativity principle; the laws of physics are the same in all IRFs, therefore, Bob should be able to use the same technique Alice did to send a superluminal signal back to Alice, and he can make it have the same velocity according $S^{\prime}$ that Alice's signal had according to $S$.

Now, by using ST (7) and the definition of specific kinematical parameter (1), let us evaluate the time of arrival of superluminal signal relative to $S$ and $S^{\prime}$, respectively.

Relative to $S$, the time arrival is given by:

$\Delta t=t_{B}-t_{A}=\frac{B-A}{u}$.(i)

Relatively to $S^{\prime}$, the time of arrival is given according to the ST (7):

$\Delta t^{\prime}=t_{B}^{\prime}-t_{A}^{\prime}=\eta\left(t_{B}-\frac{v B}{\vartheta^{2}(v)}\right)-\eta\left(t_{A}-\frac{v A}{\vartheta^{2}(v)}\right)=\eta\left(1-\frac{v u}{\vartheta^{2}(v)}\right) \Delta t$, (ii)

with $\eta=1 / \sqrt{1-v^{2} / \vartheta^{2}(v)}$. 
Remark: since in $(i)$, we have $\Delta t>0$ and in (ii), we have $v u<\vartheta^{2}(v)$ because Alice and Bob (when they were coincident at $O \equiv O^{\prime}$ ) could have arranged in advance to respect the inequality $v u<\vartheta^{2}(v)$ in order to have a superluminal communication without violating convention causality and relativity principle. Therefore, according to Alice's and Bob's arrangement we can always choose $v u<\vartheta^{2}(v)$ such that $\left(1-v u / \vartheta^{2}(v)\right)>0$, consequently $\Delta t^{\prime}>0$. From all that, we arrive at the following conclusion:

$$
\text { If } \Delta t>0 \text { in } S \Rightarrow \Delta t^{\prime}>0 \text { in } S^{\prime} \text {. }
$$

That is to say the time ordering (chronology) is perfectly respected and the superluminal causality is in fact an ordinary thing in the context of superluminal relativistic kinematics.

\section{IV.2. Superluminal relativistic dynamics}

After having derived the expected laws of superluminal relativistic kinematics, now, we focus our attention on the formulation of superluminal relativistic dynamics. As we have already mentioned it explicitly or implicitly, in the superluminal formalism, all superluminal physical equations should be invariant under STs during any transition from an IRF to another. Therefore, such equations should be defined in superluminal space-time. For example, the invariance of superluminal quadratic form (2) is the first superluminal invariance. Thus, basing on such a central idea, we will show the existence of the following physical quantities: superluminal momentumenergy four-vector; superluminal three-dimensional momentum; superluminal (total kinetic) energy; superluminal momentum-energy relation and superluminal force four-vector.

\section{IV.2.1. Superluminal momentum-energy four-vector}

By definition, the combination of superluminal three-dimensional momentum $\mathbf{p}\left(p_{x}, p_{y}, p_{z}\right)$ and the superluminal (total kinetic) energy $E$ via SKP forms, in superluminal space-time, a superluminal momentum-energy four-vector

$$
\mathbf{P}=\left(\mathbf{p}, i \frac{\mathscr{E}}{\vartheta(v)}\right)
$$

that should characterize any material point moving at superluminal velocity. This superluminal momentum-energy four-vector (30) should be invariant under STs during any transition from an IRF to another. Specifically, for an IRF $S^{\prime}$ in straight-line uniform motion at superluminal velocity $\mathbf{v}$ of magnitude $v$ relative to $S$ along the $x$-axis, the superluminal transformations of $\mathbf{P}$ are:

$$
S \rightarrow S^{\prime}:\left\{\begin{array}{l}
p_{x^{\prime}}^{\prime}=\eta\left(p_{x}-\varepsilon \frac{\mathscr{E}}{\vartheta(v)}\right) \\
p_{y^{\prime}}^{\prime}=p_{y} \\
p_{z^{\prime}}^{\prime}=p_{z} \\
\frac{\mathscr{E}^{\prime}}{\vartheta(v)}=\eta\left(\frac{\mathscr{E}}{\vartheta(v)}-\varepsilon p_{x}\right)
\end{array}\right.
$$

and

$$
S^{\prime} \rightarrow S:\left\{\begin{array}{l}
p_{x}=\eta\left(p_{x^{\prime}}^{\prime}+\varepsilon \frac{\mathscr{E}^{\prime}}{\vartheta(v)}\right) \\
p_{y}=p_{y^{\prime}}^{\prime} \\
p_{z}=p_{z^{\prime}}^{\prime} \\
\frac{\mathscr{E}}{\vartheta(v)}=\eta\left(\frac{\mathscr{E}^{\prime}}{\vartheta(v)}+\varepsilon p_{x^{\prime}}^{\prime}\right)
\end{array}\right.
$$


To be sure that we have really defined a superluminal momentum-energy four-vector, it suffices to show from (31) or (32) the following superluminal invariance

$$
p_{x^{\prime 2}}^{\prime}+p_{y^{\prime 2}}^{\prime}+p_{z^{\prime 2}}^{\prime}-\frac{\mathscr{E}^{\prime 2}}{\vartheta^{2}(v)}=p_{x}^{2}+p_{y}^{2}+p_{z}^{2}-\frac{E^{2}}{\vartheta^{2}(v)},
$$

or more compactly

$$
P^{\prime 2}-\frac{E^{\prime 2}}{\vartheta^{2}(v)}=P^{2}-\frac{E^{2}}{\vartheta^{2}(v)}
$$

\section{IV.2.2. Superluminal three-dimensional momentum and superluminal (total kinetic) energy}

If we now apply ST (32) to a material point of mass $m$ in its proper superluminal frame $S^{\prime}$ (where the material point is at relative rest), in the observer's frame $S$ (where the same material point is seen to move at superluminal velocity), we obtain after a simple calculation the following expected superluminal three-dimensional momentum and superluminal (total kinetic) energy

$$
p=\frac{\mathscr{E}}{\vartheta^{2}(v)} v
$$

and

$$
\mathscr{E}=\eta \mathscr{E} 0
$$

where $\eta=1 / \sqrt{1-v^{2} / \vartheta^{2}(v)}, c \leq v<\vartheta(v)$ and $\mathscr{E}_{0}=\mathscr{E}^{\prime}=m c^{2}$ is the rest mass energy of the material point (the subscript 0 indicating, henceforth, quantity of the physical system referred to a comoving IRF.

\section{IV.2.3. Derivation of superluminal kinetic energy}

Concerning the superluminal kinetic energy $\mathscr{E}_{K}$ of a material body moving at a superluminal velocity, is explicitly defined by the expression

$$
\mathscr{E}_{K}=\mathscr{E}-\mathscr{E}_{0}=\mathscr{E}_{0}(\eta-1)
$$

Rigorously, the formula (37) may be derived as follows. Firstly, recall that in classical mechanics the work done accelerating a particle during the infinitesimal time interval $d t$ is given by the $d o t$ product of force $\mathbf{f}$ and displacement $d \mathbf{r}$ :

$$
\mathbf{f} \cdot d \mathbf{r}=\mathbf{f} \cdot \mathbf{v} d t=\frac{d \mathbf{p}}{d t} \cdot \mathbf{v} d t=\mathbf{v} \cdot d p=\mathbf{v} \cdot d(m \mathbf{v}),
$$

where we have assumed the well-known classical 3D-momentum expression $\mathbf{p}=m \mathbf{v}$. Applying the dot product rule we see that:

$$
d(\mathbf{v} \cdot \mathbf{v})=(d \mathbf{v}) \cdot \mathbf{v}+\mathbf{v} \cdot(d \mathbf{v})=2(\mathbf{v} \cdot d \mathbf{v}) .
$$

Therefore (assuming constant mass so that $d m=0$ ), the following can be seen:

$$
\mathbf{v} \cdot d(m \mathbf{v})=\frac{m}{2} d(v \cdot v)=\frac{m}{2} d v^{2}=d\left(\frac{m v^{2}}{2}\right) .
$$

Since this is a total differential (that is, it only depends on the final state, not how the particle got there), we can integrate it and call the result kinetic energy:

$$
E_{K}=\int \mathbf{F} \cdot d \mathbf{r}=\int \mathbf{v} \cdot d(m \mathbf{v})=\int d\left(\frac{m v^{2}}{2}\right)=\frac{m v^{2}}{2} .
$$


Classically, this equation states that the kinetic energy $E_{K}$ is equal to the integral of the dot product of the velocity $v$ of a body and the infinitesimal change of the body's momentum $\mathbf{p}$. It is assumed that the body starts with no kinetic energy when it is at (relative) rest.

Superluminal Kinetic Energy: In superluminal relativistic dynamics, that is when the material object evolving in superluminal space-time at superluminal velocity, we must change the expression for linear momentum. Using $\mathscr{E}_{0}$ for rest (mass) energy, $\mathbf{v}$ with magnitude $v \geq c$ for the superluminal object's velocity vector, we assume according to the superluminal formula (35) for linear momentum $p=\eta \vartheta^{-2}(v) \mathscr{E}_{0} v$ where $\eta=1 / \sqrt{1-v^{2} / \vartheta^{2}(v)}$ and $\mathscr{E}_{0}=m c^{2}$. Hence, integrating by parts gives:

$$
\begin{aligned}
\mathscr{E}_{K} & =\int \mathbf{v} \cdot d \mathbf{p}=\int \mathbf{v} \cdot d\left(\eta \vartheta^{-2}(v) \mathscr{E}_{0} \mathbf{v}\right)=\eta \vartheta^{-2}(v) \mathscr{E}_{0} \mathbf{v} \cdot \mathbf{v}-\int \eta \vartheta^{-2}(v) E_{0} v \cdot d v \\
& =\eta \vartheta^{-2}(v) \mathscr{E}_{0} v^{2}-\int \eta \vartheta^{-2}(v) \mathscr{E}_{0} v \cdot d v=\eta \vartheta^{-2}(v) \mathscr{E}_{0} v^{2}-\frac{\mathscr{E}_{0}}{2} \int \eta \vartheta^{-2}(v) d\left(v^{2}\right) .
\end{aligned}
$$

Remembering that $\vartheta(v)$ is conceptually defined as a constant speed in all the IRFs and $\eta=1 / \sqrt{1-v^{2} / \vartheta^{2}(v)}$, hence from above we get:

$$
\begin{aligned}
\mathscr{E}_{K} & =\eta \vartheta^{-2}(v) \mathscr{E}_{0} v^{2}-\frac{-E_{0}}{2} \int \eta d\left(1-v^{2} / \vartheta^{2}(v)\right) \\
& =\eta \vartheta^{-2}(v) \mathscr{E}_{0} v^{2}+\mathscr{E}_{0} \sqrt{1-v^{2} / \vartheta^{2}(v)}+C .
\end{aligned}
$$

The integration constant $C$ may be identified as $-\mathscr{E}_{0}$ that is when the material body in question is in a state of (relative) rest $\mathscr{E}_{K}=0$, since according to (1), we have $\vartheta(v)=c$ for $v=0$, thus finally we get

$$
\mathscr{E}_{K}=\mathscr{E}_{0}(\eta-1)
$$

\section{IV.2.4. Explicit expression of SKP}

It is important to note that in the context of superluminal relativistic dynamics, we call the quantity $\varepsilon=v / \vartheta(v)$ fractional superluminal (relative) velocity, where $v$ should be expressed in units of $c$ with $c \leq v<\vartheta(v)$. Practically, it is very useful to use $\varepsilon$ instead of $v$ in order to perform the calculations more easily. Our aim in this subsection is the derivation of an explicit expression for SKP from the Eta-factor $\eta$ as a function of $\varepsilon$. To this end, we have

$$
\eta=\frac{1}{\sqrt{1-\varepsilon^{2}}}
$$

Basically, Eta-factor may be determined from (37) by the formula $\eta=1+\mathscr{E}_{K} / \mathscr{E}_{0}$. Finally, from (38) we find the very expected expression of SKP as a function of $v$ :

$$
\vartheta(v)=\frac{\eta v}{\sqrt{\eta^{2}-1}}
$$

As we will see, the expression (39) allows us to evaluate SKP for any well determined numerical values of $\eta$ and $v$, respectively. For instance, in order to examine, numerically, the behavior of SKP, we have selected and listed in Table1, certain idealized numerical values to illustrate the constancy of $\vartheta(v)$. 
Table 1. Set of idealized numerical values for $\varepsilon$ and $v$ is proposed and from where $\eta$ and $\vartheta(v)$ are computed and listed.

\begin{tabular}{cccc}
\hline \hline$\varepsilon$ & $\eta$ & $v(c)$ & $\vartheta(v)$ \\
\hline \hline 0.15 & 1.011443 & 1.5 & 10 \\
0.25 & 1.032795 & 2.5 & 10 \\
0.35 & 1.067521 & 3.5 & 10 \\
0.45 & 1.119785 & 4.5 & 10 \\
0.55 & 1.197368 & 5.5 & 10 \\
0.65 & 1.315903 & 6.5 & 10 \\
0.75 & 1.511857 & 7.5 & 10 \\
0.85 & 1.898315 & 8.5 & 10 \\
0.95 & 3.202563 & 9.5 & 10 \\
\hline \hline
\end{tabular}

As we can remark it from Table 1, SKP as a function of superluminal relative velocity $\mathrm{v}$ stayed constant monotonically for different idealized numerical values for $\eta$ and $v$. This constancy may be seen as a numerical confirmation of the relation (17).

\section{IV.2.5. Superluminal momentum-energy relation}

By combining the formulae (35) and (36), we get the very expected superluminal momentumenergy relation

$$
\mathscr{E}^{2}=\vartheta^{2}(v) \mathbf{p}^{2}+\mathscr{E}_{0}^{2},
$$

or

$$
\mathscr{E}=\sqrt{\vartheta^{2}(v) \mathbf{p}^{2}+\mathscr{E}_{0}^{2}}
$$

Therefore, in superluminal relativistic dynamics, the superluminal momentum-energy relation (40) or (41) is a superluminal formula relating any physical object's rest mass energy, total energy and momentum. -As a direct result, the above superluminal formulae enable as to consider the photon and the tachyon as ordinary particles with nonzero (relative) rest mass. Consequently, the old notion of zero rest mass for the first and imaginary (rest) mass for the second becomes not only meaningless but simply absurd! Because according to the principle of KLs there is a set of reference systems called luminal IRFs relative to which photons behave as ordinary particles; the same thing for the tachyons, i.e., the set of superluminal IRFs allows the tachyons to behave as ordinary particles ${ }^{2}$.

\section{IV.2.6. Superluminal Lagrangian and Hamiltonian}

Lagrangian and Hamiltonian as functions are so important in mechanics that's why must be adjusted in light of the new concepts presented in the superluminal formalism. We can extend the Lagrangian formalism into the realm of the superluminal relativistic mechanics in the following way.

\footnotetext{
${ }^{2}$ By ordinary particles, we mean bradyons,i.e., particles that travel at subluminal velocities. The term 'bradyon', from Greek: $\beta \rho \alpha \delta$ v́ (bradys, 'slow'), was initially coined to contrast with tachyon, from Greek: $\tau \alpha \chi v \varsigma$ (tachys, 'rapid').
} 
Firstly, for a single subrelativistic ${ }^{3}$ particle moving in a velocity-independent potential, the 3D-momentum may be classically written as

$$
\mathbf{p}=\frac{\partial \mathscr{L}}{\partial v} \frac{\mathbf{v}}{v}
$$

According to Eq. (35), the superluminal 3D-momentum is $\mathbf{p}=\eta \vartheta^{-2}(v) \mathscr{E}_{0} \mathbf{v}$, with $\eta=$ $1 / \sqrt{1-v^{2} / \vartheta^{2}(v)}$ and $\mathscr{E}_{0}=m c^{2}$.

We now require that the expected superluminal Lagrangian, when differentiated with respect to $v$ as in Eq.(42), yields the superluminal 3D-momentum given by Eq.(35).

$$
\frac{\partial \mathscr{L}}{\partial v} \frac{\mathbf{v}}{v}=\eta \vartheta^{-2}(v) \mathscr{E}_{0} \mathbf{v}
$$

This requirement involves only the superluminal velocity of the particle, so we expected that the velocity-independent part of the superluminal Lagrangian is unchanged from the classical case.

The velocity-dependent part, however, may no longer be explicitly equal to the superluminal kinetic energy. We therefore write

$$
\mathscr{L}=T-U,
$$

where $U \equiv U(r)$ with $r=\sqrt{x^{2}+y^{2}+z^{2}}$ and $T \equiv T(v)$ with $v=\sqrt{v_{x}^{2}+v_{y}^{2}+v_{z}^{2}}$. Furthermore, the function $T$ must satisfy the relation

$$
\frac{\partial T}{\partial v}=\eta \vartheta^{-2}(v) E_{0} v
$$

Recalling that $\vartheta(v)$ is conceptually defined as a constant speed in all the IRFs, thus it can be easily verified that a suitable expression for $T$ (apart from a possible integration constant that can be suppressed) is

$$
T=-\eta^{-1} \mathscr{E} 0 .
$$

Consequently, by substituting (46) in (44), we obtain the expected superluminal Lagrangian

$$
\mathscr{L}=-\eta^{-1} \mathscr{E}_{0}-U
$$

or more explicitly

$$
\mathscr{L}=-\mathscr{E}_{0} \sqrt{1-v^{2} / \vartheta^{2}(v)}-U, \quad \mathscr{E}_{0}=m c^{2} .
$$

The equations of superluminal motion should be obtained in the standard way from Lagrange's equations. Notice that the Lagrangian should be given according to the superluminal expression for the kinetic energy (37).

The Hamiltonian can be evaluated from the following expression

$$
H=\mathbf{p} \cdot \mathbf{v}-\mathscr{L},
$$

By using Eqs. (35), (48), (36), (40) and (41), the above expression becomes

\footnotetext{
${ }^{3}$ Term/adjective describing a velocity (and associated properties) that is considerably less than the light speed (in vacuum) such that relativistic effects may be ignored.
} 


$$
\begin{aligned}
H & =\mathbf{p} \cdot \frac{\mathbf{p}}{\eta \vartheta^{-2}(v) \mathscr{E}_{0}}+\mathscr{E}_{0} \sqrt{1-v^{2} / \vartheta^{2}(v)}+U=\frac{\mathbf{p}^{2}}{\eta \vartheta^{-2}(v) \mathscr{E}_{0}}+\eta^{-1} \mathscr{E}_{0}+U \\
& =\frac{\mathbf{p}^{2}+\vartheta^{-2}(v) \mathscr{E}_{0}^{2}}{\eta \vartheta^{-2}(v) \mathscr{E}_{0}}+U=\frac{\mathbf{p}^{2}+\vartheta^{-2}(v) \mathscr{E}_{0}^{2}}{\vartheta^{-2}(v) \mathscr{E}^{2}}+U \\
& =\frac{\left(\vartheta^{2}(v) \mathbf{p}^{2}+\mathscr{E}_{0}^{2}\right) \vartheta^{-2}(v)}{\vartheta^{-2}(v) \sqrt{\vartheta^{2}(v) \mathbf{p}^{2}+\mathscr{E}_{0}^{2}}}+U=\sqrt{\vartheta^{2}(v) \mathbf{p}^{2}+\mathscr{E}_{0}^{2}}+U=\mathscr{E}+U
\end{aligned}
$$

The superluminal Hamiltonian is equal to the superluminal (total kinetic) energy (39) plus the potential energy.

\section{IV.2.7. Superluminal force four-vector}

In classical dynamics, to use Newton's second law of motion, the force must be defined as the rate of change of momentum with respect to the same time coordinate. That is, it requires the 3D-force vector. In superluminal relativistic dynamics, if a material point is characterized by the superluminal velocity vector $\mathbf{u}\left(u_{x}, u_{y}, u_{z}\right)$ of magnitude $u$, we can transform the superluminal 3D-force vector from the material point's comoving IRF into the observer's IRF. This yields a four-vector called 'the superluminal force four-vector'. It is, by definition, the rate of change of the superluminal momentum-energy four-vector with respect to material point's proper time. Hence, the superluminal invariance version of this superluminal force four-vector is

$$
\mathbf{F}=\frac{d \mathbf{P}}{d t^{*}}=\left(\eta \mathbf{f}, i \frac{\eta(\mathbf{f} \cdot \mathbf{u})}{\vartheta(u)}\right)
$$

where $\eta=1 / \sqrt{1-u^{2} / \vartheta^{2}(u)}$, and $\mathbf{f}$ and $\mathbf{u}$ are, respectively, the superluminal 3D-force vector and superluminal velocity vector of the moving material point.

\section{IV.2.8. Transformations of superluminal force four-vector}

Like for the case of superluminal velocity four-vector, that is, we use the fact that the components of $\mathbf{F}$ should transform from a superluminal IRF to another IRF via STs:

$$
\mathbf{F}^{\prime}=\mathscr{H} F, \quad \mathbf{F}=\left(\eta \mathbf{f}, i \frac{\eta(\mathbf{f} \cdot \mathbf{u})}{\vartheta(u)}\right), \quad \mathbf{F}^{\prime}=\left(\eta^{\prime} \mathbf{f}^{\prime}, i \frac{\eta^{\prime}\left(\mathbf{f}^{\prime} \cdot \mathbf{u}^{\prime}\right)}{\vartheta\left(u^{\prime}\right)}\right)
$$

where $\mathscr{H}$ is the matrix of ST (7), $\eta=1 / \sqrt{1-u^{2} / \vartheta^{2}(u)}, \eta^{\prime}=1 / \sqrt{1-u^{\prime 2} / \vartheta^{2}\left(u^{\prime}\right)}$ and $\mathbf{u}$ being the superluminal velocity vector of the material point relative to IRF $S$, and $\mathbf{u}^{\prime}$ the superluminal velocity vector of the same material point relative to IRF $S^{\prime}$. Let $\mathbf{v}$ of magnitude $v$ be the 
superluminal velocity vector of $S^{\prime}$ with respect to $S$ along the $x$-axis. Thus, we have

$$
S \rightarrow S^{\prime}:\left\{\begin{array}{l}
\eta^{\prime} f_{x^{\prime}}^{\prime}=\eta_{(v)}\left(\eta f_{x}-\varepsilon \frac{\eta(\mathbf{f} \cdot \mathbf{u})}{\vartheta(v)}\right) \\
\eta^{\prime} f_{y^{\prime}}^{\prime}=\eta f_{y} \\
\eta^{\prime} f_{z^{\prime}}^{\prime}=\eta f_{z} \\
\frac{\eta^{\prime}\left(\mathbf{f}^{\prime} \cdot \mathbf{u}^{\prime}\right)}{\vartheta\left(u^{\prime}\right)}=\eta_{(v)}\left(\frac{\eta(\mathbf{f} \cdot \mathbf{u})}{\vartheta(v)}-\varepsilon \eta f_{x}\right)
\end{array}\right.
$$

where

$$
\eta_{(v)}=1 / \sqrt{1-v^{2} / \vartheta^{2}(v)}, \quad \varepsilon=v / \vartheta(v), \quad v \geq c
$$

Recall, during the derivation of the transformation of superluminal velocity four-vector, we have found some relation between the superluminal factors $\eta, \eta^{\prime}$ and $\eta_{(v)}$, defined by the expression (23), which should be used here. Furthermore, by taking into account the principle of KLs or more precisely the fact that all the IRFs belonging to the same KL are equivalent, thus we can consider the case $\vartheta(v)=\vartheta(u)=\vartheta\left(u^{\prime}\right)$ and deduce the expected transformations of superluminal force four-vector, that is, the explicit transformations of components of superluminal $3 \mathrm{D}$-force vector $\mathbf{f}$ and the instantaneous superluminal power $(\mathbf{f} \cdot \mathbf{u})$ :

$$
S \rightarrow S^{\prime}:\left\{\begin{array}{l}
f_{x^{\prime}}^{\prime}=\left(1-\frac{u_{x} v}{\vartheta^{2}(v)}\right)^{-1}\left(f_{x}-\frac{(\mathbf{f} \cdot \mathbf{u}) v}{\vartheta^{2}(v)}\right) \\
f_{y^{\prime}}^{\prime}=\left(1-\frac{v^{2}}{\vartheta^{2}(v)}\right)^{1 / 2}\left(1-\frac{u_{x} v}{\vartheta^{2}(v)}\right)^{-1} f_{y} \\
f_{z^{\prime}}^{\prime}=\left(1-\frac{v^{2}}{\vartheta^{2}(v)}\right)^{1 / 2}\left(1-\frac{u_{x} v}{\vartheta^{2}(v)}\right)^{-1} f_{z} \\
\left(\mathbf{f}^{\prime} \cdot \mathbf{u}^{\prime}\right)=\left(1-\frac{u_{x} v}{\vartheta^{2}(v)}\right)^{-1}\left((\mathbf{f} \cdot \mathbf{u})-v f_{x}\right)
\end{array}\right.
$$

and

$$
S^{\prime} \rightarrow S:\left\{\begin{array}{l}
f_{x}=\left(1+\frac{u_{x^{\prime}}^{\prime} v}{\vartheta^{2}(v)}\right)^{-1}\left(f_{x^{\prime}}^{\prime}+\frac{\left(\mathbf{f}^{\prime} \cdot \mathbf{u}^{\prime}\right) v}{\vartheta^{2}(v)}\right) \\
f_{y}=\left(1-\frac{v^{2}}{\vartheta^{2}(v)}\right)^{1 / 2}\left(1+\frac{u_{x^{\prime}}^{\prime} v}{\vartheta^{2}(v)}\right)^{-1} f_{y^{\prime}}^{\prime} \\
f_{z}=\left(1-\frac{v^{2}}{\vartheta^{2}(v)}\right)^{1 / 2}\left(1+\frac{u_{x^{\prime}}^{\prime} v}{\vartheta^{2}(v)}\right)^{-1} f_{z^{\prime}}^{\prime} \\
(\mathbf{f} \cdot \mathbf{u})=\left(1+\frac{u_{x^{\prime}}^{\prime} v}{\vartheta^{2}(v)}\right)^{-1}\left(\left(\mathbf{f}^{\prime} \cdot \mathbf{u}^{\prime}\right)+v f_{x^{\prime}}^{\prime}\right)
\end{array}\right.
$$

Now, we can say that we have really formulated the two principal parts of the SupRelMec, namely, the superluminal relativistic kinematics and dynamics. In this sense, we can affirm that SRT is Superluminalized.

\section{CONCLUSION}

We have derived new superluminal spatio-temporal transformations based on the idea that, conceptually and kinematically, each subluminal $(0 \leq v<c)$, luminal $(v=c)$ and/or superluminal 
inertial reference frame $v>c$ has, in addition to its relative velocity $v$, its proper specific kinematical parameter $\vartheta(v)$, which having the physical dimensions of a constant speed. Basing on such a derivation, we have formulated the superluminal relativistic mechanics, which is in fact a pure superluminalization of SRT.

\section{REFERENCES}

[1] A. Einstein, Ann. Phys. (Leipzig) 17 (1905) 891

[2] O. Bilaniuk and E. Sudarshan, Physics Today, 22 (1969) 43

[3] E. Recami, Relativity Theory and its Generalization, in Astrophysics, Quanta and Relativity Theory (Moscow, Izdatelsvo Mir, 1982) pp. 53-128.

[4] R. Mignani, Lett. Nuovo Cimento, 13 (1975) 134

[5] C. E. Patty, Nuovo Cimento, 70B (1982) 65

[6] E. Recami, G. D. Maccarrone, Lett. Nuovo Cimento, 28, 151 (1980)

[7] L. Parker, Phys. Rev. 188 (1969) 2287

[8] L. Marchildon, A. F. Antippa and A. E. Everett, Phys. Rev. D 27 (1983) 1740

[9] R. I. Sutherland and J. R. Shepanski, Phys. Rev. D 33 (1986) 2896

[10] A. Einstein, Ann. Phys. 328 (1907) 371

[11] G. Nimtz and A. Enders, J. Phys. I (France) 2 (1992) 1693

[12] A. Ender and G. Nimtz, Phys. Rev. B 47 (1993) 9605

[13] A. Ender and G. Nimtz, J. Phys. I (France) 3 (1993) 1089

[14] G. Nimtz, A. Enders and H. Spieker, J. Phys. I (France) 4, 565 (1994)

[15] G. Nimtz and A. Haibel, arXiv:physics/0104063v1 (2001)

[16] G. Nimtz and W. Heitmann, Prog. Quantum Electron. 21 (1997) 81

[17] L. J. Wang, A. Kuzmich and A. Dogariu, Nature 406 (2000) 277

[18] T. J. Pearson et al, Nature 290 (1981) 365

[19] A. Brunthaler et al, A\&A 357 (2000) L 45

[20] I. F. Mirabel and L. F. Rodriguez, Nature 371 (1994) 46

[21] S. J. Newell, M. A. Garrett and R. E. Spencer, MNRAS 293 (1998) L17

[22] U.S. Inan et al, Geophys. Res. Lett. 23 (1996) 133

[23] A. Einstein, Jaharb. Radioakt. Elektron. 4 (1907) 411 